\title{
Separating the efflux and influx components of net nitrate uptake by Synechococcus $\mathbf{R} 2$ under steady-state conditions
}

\author{
Georgia Shearer, ${ }^{*}$ Julie D. Schneider and Daniel H. Kohl \\ Biology Department, Washington University, St Louis, MO 63130, USA
}

(Received 2 July 1990; revised 3 December 1990; accepted 17 January 1991)

\begin{abstract}
$\mathrm{N}$ isotopic discrimination was used to determine the ratio of influx to efflux in experiments in which $\mathrm{NO}_{3}^{-}$reduction was decreased (and also net $\mathrm{NO}_{3}^{-}$uptake, since under steady-state conditions, net $\mathrm{NO}_{3}^{-}$uptake is equal to the rate of reduction) by tungstate. Exponential growth rate constants for the cyanobacterium Synechococcus Sp. R2 grown in media containing $0.18 \mu \mathrm{M}$-molybdate decreased linearly $\left(0.040\right.$ to $\left.0.013 \mathrm{~h}^{-1}\right)$ with increasing tungstate concentration ( 0 to $0.2 \mathrm{mM}$ ). Values of the overall, observed $N$ isotope effect $\left(\beta_{\text {obs }}\right.$, the ratio of the rate of reaction of ${ }^{14} \mathrm{~N}$ - to ${ }^{15} \mathrm{~N}$-bearing molecules, normalized to adjust for unequal concentrations) varied inversely and were linear with exponential growth rate. At high tungstate concentration, when the exponential growth rate approached zero (as a result of negligible $\mathrm{NO}_{3}^{-}$reduction), $\beta_{\text {obs }}$ approached 1.0197. In the absence of tungstate, $\beta_{\text {obs }}$ was equal to 1.0037. From this value, we calculated the ratio of efflux to influx to be about $0 \cdot 19$. That is, in the absence of tungstate, more than $80 \%$ of the $\mathrm{NO}_{3}^{-}$entering the cell underwent reduction with less than $20 \%$ released to the medium from the cell. This approach can be used to distinguish changes in influx from changes in metabolic steps in response to factors which affect net uptake rate.
\end{abstract}

\section{Introduction}

Considerable information is available on factors affecting assimilation of $\mathrm{NO}_{3}^{-}$by Synechococcus sp. R2 (PCC 7942). In this organism, reduction of $\mathrm{NO}_{3}^{-}$and $\mathrm{NO}_{2}^{-}$, as well as $\mathrm{CO}_{2}$, requires the photochemically generated electron donor reduced ferredoxin (Candau et al., 1976; Lara \& Romero, 1986). For this reason, and also because active $\mathrm{CO}_{2}$ fixation is independently required, presumably to provide carbon skeletons for $\mathrm{N}$ assimilation (Romero et al., 1985), $\mathrm{NO}_{3}^{-}$assimilation is dependent on light intensity (Lara \& Romero, 1986). Utilization of $\mathrm{NO}_{3}^{-}$is inhibited by $\mathrm{NH}_{4}^{+}$(Flores et al., 1980; Herrero et al., 1981) as well as by DL-glyceraldehyde (DLG), an inhibitor of $\mathrm{CO}_{2}$ fixation (Romero et al., 1985). Shortterm inhibition by $\mathrm{NH}_{4}^{+}$of $\mathrm{NO}_{3}^{-}$utilization occurs without effect on nitrate reductase (NR) activity (Flores et al., 1980). However, in the long-term, NR activity is inhibited (Herrero et al., 1981). Restoration of activity is inhibited by rifampicin, indicating transcriptional control of NR synthesis by $\mathrm{NH}_{4}^{+}$.

Negative effectors may act either by decreasing $\mathrm{NO}_{3}^{-}$ influx or by decreasing the rate of metabolic steps. In short-term experiments Lara et al. (1987) provided the

\footnotetext{
Abbreviations: $\beta_{\text {obs, }}$ overall, observed $\mathrm{N}$ isotope effect; DLG, DL-glyceraldehyde; NR, nitrate reductase.
}

following evidence that $\mathrm{NH}_{4}^{+}$and DLG act on the uptake step. Molybdenum is required for the synthesis of active NR (Candau, 1979). Because tungsten is a structural analogue of molybdenum, substitution of tungstate for molybdate in the growth medium results in the synthesis of inactive NR. Lara et al. (1987) demonstrated active transport of $\mathrm{NO}_{3}^{-}$in cells with tungstate-inactivated NR. Intracellular $\mathrm{NO}_{3}^{-}$concentrations in such cells were four to eight times higher than in the medium $60 \mathrm{~s}$ after exposure to $\mathrm{NO}_{3}^{-}$. No accumulation occurred in the presence of $\mathrm{NH}_{4}^{+}$or DLG, indicating that these inhibitors act on the uptake system. However, long-term exposure to $\mathrm{NH}_{4}^{+}$must have an effect beyond the uptake step, since active NR (the synthesis of which does not require the presence of $\left.\mathrm{NO}_{3}^{-}\right)$is not synthesized under these conditions (Herrero et al., 1981).

It would be of considerable interest to determine the relative degree to which negative (or positive) effectors of $\mathrm{NO}_{3}^{-}$utilization exert their effect through changes in influx or metabolism. Present methods do not permit such a determination under steady-state conditions. Measurements of influx, as opposed to net uptake rate, usually involve short-term experiments in which the rate of transport of labelled substrate (or labelled substrate analogue) from the medium into cells is determined. Such measurements provide transient values for influx, which may be different from influx after a steady-state 
has been established. Under steady-state conditions, $\mathrm{NO}_{3}^{-}$influx is equal to the rate of $\mathrm{NO}_{3}^{-}$metabolism plus efflux, with the net uptake rate being equal to the rate of $\mathrm{NO}_{3}^{-}$metabolism (i.e., the rate of metabolism is equal to influx minus efflux). Therefore, if it were possible to measure the ratio of efflux to influx, this ratio, together with a measured value for net uptake rate would permit calculation of the absolute values of influx and efflux. In this paper we report the positive result of a feasibility test of this approach to separating the influx and efflux components of net $\mathrm{NO}_{3}^{-}$uptake.

\section{Methods}

Synechocococcus sp. R2 was obtained from L. Sherman (Purdue University, West Lafayette, IN, USA). Cultures were maintained on $1.5 \%$ (w/v) agar BG-11 medium (Rippka et al., 1979), with $5 \mathrm{~mm}$ $\mathrm{NaNO}_{3}$. Experimental cultures were grown in $800 \mathrm{ml}$ or $100 \mathrm{ml}$ of liquid medium BG-11 containing $2 \mathrm{~mm}-$ or $5 \mathrm{~mm}-\mathrm{NaNO}_{3}$. Liquid cultures were kept in an illuminated (two $40 \mathrm{~W}$ daylight fluorescent strips at a distance of $21 \mathrm{~cm}$ ) water-bath at $30^{\circ} \mathrm{C}$, and bubbled with a mixture of $5 \%(\mathrm{v} / \mathrm{v}) \mathrm{CO}_{2}$ in air. Growth was followed by measuring the optical density at $750 \mathrm{~nm}$.

After growth for an appropriate time, samples were collected and cells removed by centrifugation for $10 \mathrm{~min}$ at $10000 \mathrm{~g}$. In order to determine the relationship between $\mathrm{OD}_{750}$ and protein concentration, cells at various growth stages were washed twice with $100 \mathrm{~mm}$ $\mathrm{NaHCO}_{3}$ and $0.8 \mathrm{~mm}-\mathrm{MgSO}_{4}$, adjusted to $\mathrm{pH} 7 \cdot 4$. Washed cells were subjected to Kjeldahl digestion followed by distillation of the $\mathrm{NH}_{4}^{+}$in the digest into boric acid containing a mixed indicator, and titration with standard $\mathrm{HCl}$ (Shearer \& Kohl, 1986). Protein was assumed to contain $16 \%(\mathrm{w} / \mathrm{w}) \mathrm{N}$ (Bergersen, 1980). The correlation coefficient between $\mathrm{OD}_{750}$ and protein concentration was 0.98 , with the slope corresponding to $200 \mu \mathrm{g}$ protein (OD unit) ${ }^{-1}$. Loss of $\mathrm{NO}_{3}^{-}$from the medium was measured by steam distillation techniques (Bremner \& Keeney, 1965). Any $\mathrm{NH}_{4}^{+}$which might have been present was separated from the sample by steam distillation (in the presence of $\mathrm{MgO}$ ) prior to reducing $\mathrm{NO}_{3}^{-}$. No free $\mathrm{NH}_{4}^{+}$was detected in any of the samples. We considered it unnecessary to remove $\mathrm{NO}_{2}^{-}$prior to reducing $\mathrm{NO}_{3}^{-}$. Although $\mathrm{NO}_{2}^{-}$has been shown to accumulate when Synechococcus 6301 is incubated under conditions of $\mathrm{C}$ or $\mathrm{S}$ deficiency (Krämer \& Schmidt, 1989), it did not accumulate under the conditions we used ( $5 \% \mathrm{CO}_{2}$ in the gas stream and $300 \mu \mathrm{M}-\mathrm{MgSO}_{4}$ in the medium). After reducing $\mathrm{NO}_{3}^{-}$to $\mathrm{NH}_{4}^{+}$with Devarda's alloy, the resulting $\mathrm{NH}_{4}^{+}$ was distilled. The amount of $\mathrm{N}$ in the distillate was determined by titration. After acidification and evaporation of the distillate, the $\mathrm{N}$ derived from $\mathrm{NO}_{3}^{-}$was converted to $\mathrm{N}_{2}$ in an evacuated SprinsonRittenberg tube by reaction with $\mathrm{NaOBr}$ (Shearer \& Kohl, 1986). Isotope analysis was performed on the resulting $\mathrm{N}_{2}$ using an isotope ratio mass spectrometer equipped with dual collectors and a dual inlet system (VG Micromass 602E). The amount of $\mathrm{NO}_{3}^{-}-\mathrm{N}$ lost from the medium was equal to the amount of $\mathrm{N}$ accumulation in the cells, indicating complete recovery of added $\mathrm{N}$.

\section{Results and Discussion}

\section{The principle of using isotopic discrimination for measuring efflux/influx}

The overall, observed $\mathrm{N}$ isotope effect (isotopic fractionation factor), $\beta_{\mathrm{obs}}$, is defined as the rate of reaction of
${ }^{15} \mathrm{~N}$-bearing molecules, relative to the rate of reaction of ${ }^{14} \mathrm{~N}$-bearing molecules, where both rates are normalized to adjust for unequal concentrations of the two kinds of molecules.

$$
\beta_{\mathrm{obs}}=\frac{\mathrm{d}\left[{ }^{14} \mathrm{~N}\right] / \mathrm{d} t}{\left[{ }^{14} \mathrm{~N}\right]} / \frac{\mathrm{d}\left[{ }^{15} \mathrm{~N}\right] / \mathrm{d} t}{\left[{ }^{15} \mathrm{~N}\right]}
$$

In closed systems (systems in which substrate is consumed during the course of the reaction) experimentally determined values of $\beta_{\mathrm{obs}}$ are calculated from measurements, in competitive experiments (in competetive experiments for measuring isotope effects, both the heavy and light isotopes are present in the same vessel), of the change in the natural abundance of ${ }^{15} \mathrm{~N}$ of substrate at two times (time zero, and some later time), and the extent of reaction. Natural ${ }^{15} \mathrm{~N}$ abundance is expressed as $\delta^{15} \mathrm{~N}$, the per mil ${ }^{15} \mathrm{~N}$ excess compared to a standard (customarily atmospheric $\mathrm{N}_{2}$ ).

$$
\delta^{15} \mathrm{~N}=\frac{\frac{{ }^{15} \mathrm{~N}}{14} \text { sample }-\frac{{ }^{15} \mathrm{~N}}{{ }^{14} \mathrm{~N}} \text { standard }}{\frac{{ }^{15} \mathrm{~N}}{{ }^{14} \mathrm{~N}} \text { standard }} .1000 \%
$$

The overall isotopic fractionation factor is calculated from experimental measurements using the approximate expression below (which gives the same result within experimental error as the exact expression) (Mariotti $e t$ al., 1981).

$$
\beta_{\mathrm{obs}}=\frac{\delta^{15} \mathrm{~N}_{\mathrm{s}_{0}}-\delta^{15} \mathrm{~N}_{\mathrm{s}_{1}}}{1000 \ln f}+1
$$

where $f$ is the ratio of substrate concentration at time $t$ to substrate concentration at time zero, and the subscripts ' $\mathrm{s}_{0}$ ' and ' $\mathrm{s}_{\mathrm{t}}$ ' refer to substrate at times zero and $t$, respectively.

The magnitude of $\beta_{\text {obs }}$ depends, not only upon the intrinsic isotope effects (the isotope effect associated with a single, unidirectional step within a reaction sequence is referred to as an intrinsic isotope effect) associated with individual steps within the overall reaction sequence, but also upon the relative rates of the forward and reverse steps. This latter dependence can be illustrated by an expression for $\beta_{\text {obs }}$ derived from the scheme shown in Fig. 1. In this scheme, $k_{1}, k_{2}, k_{3}, k_{4}$ and $k_{5}$ are rate constants for influx, efflux, enzyme-substrate binding, enzyme-substrate dissociation and $\mathrm{NO}_{3}^{-}$reduction, respectively. Some of these 'rate constants' subsume other reactants needed for the step to proceed. For example, the ATP concentration is subsumed in $k_{1}$ and $k_{2}$ if it is required for transport; the concentration of reduced ferredoxin may be subsumed in $k_{5}$, depending on whether $\mathrm{NR}$ is reduced before or after $\mathrm{NO}_{3}^{-} \cdot \mathrm{NR}$ association. 




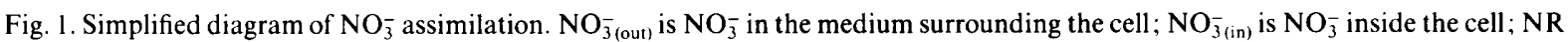
represents nitrate reductase; $\mathrm{NR} \cdot \mathrm{NO}_{3}^{-}$and $\mathrm{NR} \cdot \mathrm{NO}_{2}^{-}$represent enzyme-bound species. Rate constants for influx, efflux, enzymesubstrate association, enzyme-substrate dissociation, and reduction are indicated by $k_{1}, k_{2}, k_{3}, k_{4}$, and $k_{5}$, respectively.

The derivation of an expression for $\beta_{\text {obs }}$ based on the model shown in Fig. 1 starts with the derivation of an expression for overall velocity $(v)$. Such a derivation for an analogous five-step model has been published previously (Shearer \& Kohl, 1988), and is equivalent to the expression

$$
r=\frac{k_{1}\left[\mathrm{NO}_{3(\text { out }}^{-}\right] P Q}{1-P+P Q}
$$

where $P$ and $Q$ represent the two partition factors of the reaction sequence shown in Fig. 1.

$$
P=\frac{k_{3}[\mathrm{NR}]}{k_{2}+k_{3}[\mathrm{NR}]} \text { and } Q=\frac{k_{5}}{k_{4}+k_{5}}
$$

Thus, $P$ represents the relative amount of $\mathrm{NO}_{3(\text { (in) }}^{-}$ reacting in the forward direction, and $Q$ represents the relative amount of enzyme-bound $\mathrm{NO}_{3}^{-}$(in) reacting in the forward direction.

An expression for $\beta_{\text {obs }}$ is derived by substituting expressions for $v$ or ${ }^{15} v$ into the definition of $\beta_{\text {obs }}$ (eqn 1). The result (Shearer \& Kohl, 1988) is

$\beta_{\text {obs }}$

$$
\begin{aligned}
& =\frac{\frac{\beta_{1} \beta_{3} \beta_{5}}{\beta_{2} \beta_{4}}(1-P)(1-Q)+\frac{\beta_{1} \beta_{3}}{\beta_{2}} Q(1-P)+\beta_{1} Q P}{(1-P)(1-Q)+Q(1-P)+Q P} \\
& =\frac{\frac{\beta_{1} \beta_{3} \beta_{5}}{\beta_{2} \beta_{4}}(1-P)(1-Q)+\frac{\beta_{1} \beta_{3}}{\beta_{2}} Q(1-P)+\beta_{1} Q P}{1-P+Q P}
\end{aligned}
$$

where $\beta_{1}, \beta_{2}, \beta_{3} \beta_{4}$ and $\beta_{5}$ are intrinsic isotope effects for the indicated steps. Thus, the overall isotope effect for the reaction sequence of Fig. 1 is the weighted average of the ratios $\beta_{1} \beta_{3} \beta_{5} / \beta_{2} \beta_{4}, \beta_{1} \beta_{3} / \beta_{2}$ and $\beta_{1}$ with the weighting being related to the relative success of forward and reverse rates of the relevant steps (i.e. the partition factors $P$ and $Q$ ).

A simplifying assumption seems justified at this point. Protein-substrate complexes are almost always held together by electrostatic and hydrogen bonds, so that appreciable isotope effects on their formation and dissociation are not expected (Melander \& Saunders, 1980). Sizable $N$ isotope effects occur only on steps involving chemical reaction of the substrate. Hence $\beta_{1}$, $\beta_{2}, \beta_{3}$ and $\beta_{4}$ should have values very close to $1 \cdot 000$, and eqn 5 simplifies to

$$
\beta_{\text {obs }}=\frac{\beta_{5}(1-P)(1-Q)+Q}{1-P+Q P}
$$

Under steady-state conditions, influx - efflux $=v$, or 1 - efflux/influx $=v /$ influx. Since influx is equal to $k_{1}\left[\mathrm{NO}_{3 \text { (out) }}^{-}\right]$, the following relation holds (eqn 4)

$$
1-\frac{\text { efflux }}{\text { influx }}=\frac{P Q}{1-P+P Q}
$$

Solving this expression for $P$ and $Q$, in terms of efflux/influx, gives

$$
\begin{gathered}
P=\frac{1-\frac{\text { efflux }}{\text { influx }}}{1-\frac{\text { efflux }}{\text { influx }}(1-Q)} \text { and } \\
Q=\frac{\left(1-\frac{\text { efflux }}{\text { influx })}\right)(1-P)}{\frac{\text { efflux }}{\text { influx }} P}
\end{gathered}
$$

(The terms $P, Q$ and efflux/influx are all constrained, by definition, to lie between zero and one. $Q$ does not approach infinity as $P$ approaches zero, because in this limit, efflux/influx approaches one.)

Substituting these expressions into eqn 6 provides expressions for $\beta_{\text {obs }}$ in terms of the ratio of efflux to influx, $\beta_{5}$, and either $P$ or $Q$.

$$
\begin{aligned}
& \beta_{\text {obs }}=1+\frac{\text { efflux }}{\text { influx }}(1-Q)\left(\beta_{5}-1\right) \text { and } \\
& \beta_{\text {obs }}=\beta_{5}-\frac{1}{P}\left(1-\frac{\text { efflux }}{\text { influx }}\right)\left(\beta_{5}-1\right)
\end{aligned}
$$




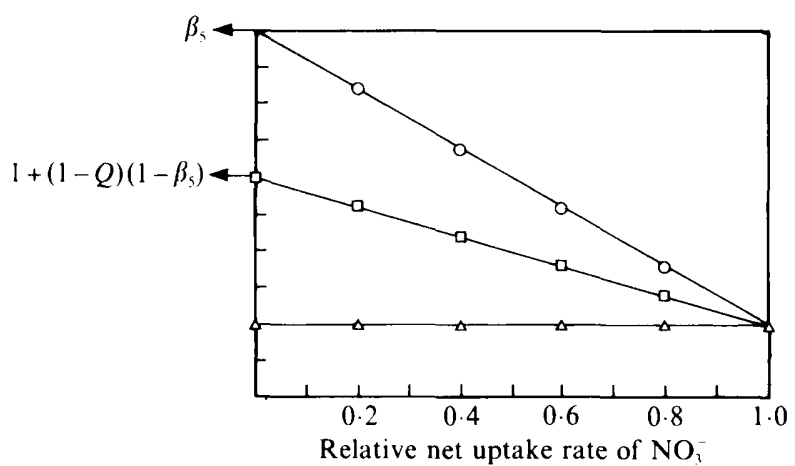

Fig. 2. Expected functional form of the relationship between the relative rate of net $\mathrm{NO}_{3}^{-}$uptake (rate in treated samples relative to rate in control samples) and $\beta_{\text {obs }}$, calculated from eqns 10 and 11 , as described in the text. $\bigcirc$, Treatment 1 - only $k_{5}$ affected; $\square$, treatment 2 - only $k_{3}[N R]$ affected; $\triangle$, treatment 3 - only $k_{1}$ affected.

Note that if either $P$ or $Q$ is constant, the relationship between efflux and $\beta_{\text {obs }}$ is linear, provided that $\beta_{5}$ is constant. $P, Q$ and $\beta_{5}$ are unknown. Therefore, the ratio of efflux to influx cannot be determined directly by experimental measurement of $\beta_{\text {obs }}$. However, the effect of a change in $v$ on $\beta_{\text {obs }}$ can be used to determine whether the change in $v$ resulted from a change in influx or metabolism. A decrease in the net rate of $\mathrm{NO}_{3}^{-}$uptake can result from a decrease in $k_{1}$ (because, for example, of a decrease in ATP concentration which is subsumed in $\left.k_{1}\right), k_{3}[\mathrm{NR}]$ (because of a decrease in NR concentration) or $k_{5}$ (which might happen if $N R$ is reduced after binding with $\mathrm{N}_{3}^{-}$and if the concentration of reduced ferredoxin is decreased). It can also result from some combination of these, but for the moment we will consider the effect of these changes on efflux/influx and $\beta_{\text {obs }}$ individually.

(1). As discussed above, if the decrease in net $\mathrm{NO}_{3}^{-}$ uptake rate is caused by a decrease in influx $\left(k_{1}\left[\mathrm{NO}_{3 \text { (out) }}^{-}\right]\right)$alone (i.e., with no concomitant decrease in $k_{3}[\mathrm{NR}]$ or $\left.k_{5}\right)$, neither partition factor $(P$ nor $Q)$ will be affected. Therefore, the decrease in influx will result in a proportional decrease in efflux (eqn 7). Since both efflux/influx and $Q$ remain constant under this condition, $\beta_{\text {obs }}$ wiil not be affected (eqn 10).

(2). If the concentration of free NR is lowered, $Q$ should not be affected, but $P$, will be decreased. As a consequence, both efflux/influx (eqn 7) and $\beta_{\text {obs }}$ will increase, with the relationship between $\beta_{\text {obs }}$ and the net rate of $\mathrm{NO}_{3}^{-}$uptake being linear (eqn 10).

(3). If $k_{5}$ is decreased, $Q$ will decrease, with a consequent increase in both efflux/influx (eqn 7) and $\beta_{\text {obs }}$ (eqn 11).

These concepts are illustrated in Fig. 2 which shows the relationship between $\beta_{\text {obs }}$ and the net uptake rate calculated from eqns 10 or 11 . The calculations were based on arbitrarily assigned values of $\beta_{5}$ and $P$ or $Q$.
Table 1. Values of $k$ (exponential growth rate constant) and $\beta_{\mathrm{obs}}$ (observed isotopic fractionation factor) during growth of Synechococcus

Synechoccous sp. was grown under standard conditions (BG-11 medium containing $1.8 \mu \mathrm{M}$-molybdate, bubbled with $5 \% \mathrm{CO}_{2}$ in air). Experiments I, II and III were replicate experiments in which the initial $\mathrm{NO}_{3}^{-}$concentration was $2 \mathrm{~mm}$. A higher initial concentration ( $5 \mathrm{~mm}$ ) was used in experiments III and IV, which are duplicate experiments.

\begin{tabular}{ccccc}
\hline \hline & $\begin{array}{c}\text { Initial } \mathrm{NO}_{3}^{-} \\
\text {Experiment }\end{array}$ & $\begin{array}{c}k \\
\left(\mathrm{~h}^{-1}\right)\end{array}$ & $\begin{array}{c}\text { No. of } \\
\text { Sompling times }\end{array}$ & $\begin{array}{c}\beta_{\text {obs }} \\
(\mathrm{mean} \pm \mathrm{SE})\end{array}$ \\
\hline I & 2.0 & 0.043 & 5 & $1.0020 \pm 0.0009$ \\
II & 2.0 & 0.035 & 4 & $1.0022 \pm 0.0009$ \\
III & 2.0 & 0.037 & 6 & $1.0014 \pm 0.0003$ \\
IV & 5.0 & 0.038 & 8 & $0.9990 \pm 0.0006$ \\
V & 5.0 & 0.032 & 4 & $0.9979 \pm 0.0008$ \\
\hline
\end{tabular}

The functional form of the relationships of Fig. 2 is independent of the values assigned.

In the discussion above, we examined the consequences of decreasing either $k_{1}, k_{3}[\mathrm{NR}]$ or $k_{5}$, in the absence of an effect of changing any of these on the others. In fact, any treatment which causes a decrease in influx may affect the partition factors $P$ and $Q$. For example, the concentration of free NR would increase as influx decreases because of the decrease in concentration of intracellular $\mathrm{NO}_{3}^{-}$, and $k_{5}$ might also increase, if reduced ferredoxin enters the reaction after $\mathrm{NO}_{3}^{-}$binds with NR, because of the decrease in concentration of the intermediate of step $5\left(\mathrm{NR} \cdot \mathrm{NO}_{3}^{-}\right)$. In either case, $P$ and $Q$ would increase, with a consequent decrease in efflux/influx (eqn 7) and $\beta_{\text {obs }}$ (eqns 10 and 11). Therefore, the effect of decreasing influx will have either no effect on $\beta_{\text {obs }}$, nor will it decrease its value. In either case, the effect of altering influx on $\beta_{\text {obs }}$ should be distinguishable from the effect of changing the rate of $\mathrm{NO}_{3}^{-}$reduction.

\section{Growth curves}

To determine the isotope effect associated with net $\mathrm{NO}_{3}^{-}$ uptake under standard conditions, and whether the magnitude of this isotope effect varied during growth, cultures supplied with $\mathrm{NaNO}_{3}$ were sampled at intervals during growth. Triplicate experiments (experiments I, II and III, Table 1) were done with an initial concentration of $2 \mathrm{mM}^{-\mathrm{NO}_{3}^{-}}$and duplicate experiments (experiments IV and V, Table 1) with an initial concentration of $5 \mathrm{~mm}-$ $\mathrm{NO}_{3}^{-}$. There was no intended difference among replicate experiments. Growth rate constants ( $k$ values) were determined from the best fit of spectrophotometric data to exponential functions and $\beta_{\text {obs }}$ was determined as described in Methods. Results are summarized in Table 1. Growth was exponential; that is, $k$ was constant 
(independent of sampling time) within individual experiments. However, from experiment to experiment $k$ varied between 0.032 and $0.043 \mathrm{~h}^{-1}$, with no significant differences between the two treatments $(2 \mathrm{~mm}$ - and $\left.5 \mathrm{mM}-\mathrm{NO}_{3}^{-}\right) . \beta_{\text {obs }}$ was also independent of sampling time within individual experiments and was very close to 1.000 in all experiments, ranging from 0.998 to 1.002 . This indicates that, under standard culture conditions, any isotope effects associated with catalytic steps were not vigorously expressed in $\beta_{\text {obs }}$. Thus, under standard conditions, the rate-limiting step preceded any isotopically sensitive step. There was no correlation between $k$ and $\beta_{\text {obs }}$, suggesting that whatever unknown variable causing differences among replicate experiments in the net rate of $\mathrm{N}$ utilization did not significantly affect the ratio of efflux to influx of $\mathrm{NO}_{3}^{-}$. However, there appeared to be a significant difference in isotopic fractionation between experiments with $2 \mathrm{~mm}$ - and $5 \mathrm{~mm}-\mathrm{NO}_{3}^{-}$. While the magnitude of $\beta_{\text {obs }}$ with both concentrations was very close to $1 \cdot 000$, a comparison of all data, pooled within each concentration, showed the difference of about $3 \%$ to be significant at the $99.9 \%$ confidence interval. We have no interpretation of this small concentration effect on $\beta_{\text {obs. }}$.

\section{Experimental manipulation designed to alter the rate of assimilation relative to efflux}

Substitution of tungstate for molybdate in the growth medium of $\mathrm{NO}_{3}^{-}$-utilizing organisms results in formation of inactive NR (Lara et al., 1987). A wild-type strain of Anacystis nidulans (Synechococcus) R2, partially inhibited with varying concentrations of tungstate (in the absence of added molydate), showed decreasing net $\mathrm{NO}_{3}^{-}$uptake rates with decreasing NR activity (Madueño et al., 1988). Variable inhibition of net $\mathrm{NO}_{3}^{-}$uptake with tungstate offers the opportunity to test one of the predictions of Fig. 2. Tungstate treatment should have no effect on influx. If reductant concentration is likewise unaffected, the only result of tungstate treatment should be to decrease the value of $P\left(=k_{3}[\mathrm{NR}] /\left\{k_{2}+k_{3}[\mathrm{NR}]\right\}\right)$, thus decreasing overall velocity, $v$ (eqn 4 ), and increasing the ratio of efflux to influx (eqn 7). Our expectation was that $\beta_{\text {obs }}$ should increase with increasing tungstate concentration and decreasing net $\mathrm{NO}_{3}^{-}$uptake rate (eqn 10).

Tungstate concentration in the growth medium was varied between 0 and $0.2 \mathrm{mM}$ in the presence of $0.18 \mu \mathrm{M}$ molybdate (the usual molybdate concentration was $1.8 \mu \mathrm{M})$. Samples were collected when the organisms had consumed about half the $\mathrm{NO}_{3}^{-}$initially present $(2 \mathrm{mM})$, estimated by spectrophotometric measurement of growth.

Fig. 3(a) shows the exponential growth rate constant, $k$, as a function of tungstate concentration. The value of $k$


Fig. 3. (a) Exponential growth rate constant, $k$, as a function of tungstate concentration; $(b)$ variation of $\beta_{\text {obs }}$ with tungstate concentration; (c) variation of $\beta_{\text {obs }}$ with $k$.

declined from 0.040 to $0.013 \mathrm{~h}^{-1}$ (corresponding to an increase in doubling time from 17.3 to $53.3 \mathrm{~h}$ ) over the concentration range of tungstate used, a decline of about $70 \%$. The relationship between and tungstate concentration and $k$ was linear $(r=0.98, p<0.005)$. With no added tungstate, the value of $k$ for a parallel experiment with the usual molybdate concentration $(1.8 \mu \mathrm{M})$ was not significantly different from the value of $k$ with $0 \cdot 18 \mu \mathrm{M}$ molydate.

Over the same tungstate concentration range, $\beta_{\text {obs }}$ increased from 1.0023 to 1.0132 (Fig. $3 b$ ), as expected from the anticipated increase in the ratio of efflux to influx, with increasing tungstate.

Provided that $Q$ was unaffected by tungstate treatment, it was expected that there should be a linear decrease in $\beta_{\text {obs }}$ with $k$ (Fig. 2). This expectation was borne out (Fig. $3 c$ ) by the high correlation coefficient for 
the relationship between $\beta_{\text {obs }}$ and $k(r=0.97, P<0.02)$. The $y$ intercept of the linear regression shown on this panel corresponds to a value of $v$ of zero. When $v$ approaches zero, efflux approaches influx under steadystate conditions; that is, the ratio of efflux to influx approaches 1. Thus the value of $\beta_{\text {obs }}$ approaches $1+(1-Q)\left(\beta_{5}-1\right)$ (eqn 10) when $v$ approaches zero. The data of Fig. 3 allow us to estimate this value at 1.0197. When the ratio of efflux to influx approaches zero, the value of $\beta_{\text {obs }}$ approaches 1.000 , since in this limit, the non-fractionating, rate-limiting step, namely influx, determines the overall rate. Since under the conditions of this experiment the relationship between $\beta_{\text {obs }}$ and $k$ was linear, it is possible to calculate the ratio of efflux to influx for any experimentally determined value of $\beta_{\text {obs }}$ from a linear interpolation between the two limits, where efllux/influx àpproaches zero or one, using eqn 10 . For example, the value of $\beta_{\mathrm{obs}}$ in the absence of tungstate, and in the presence of $0.18 \mu \mathrm{M}$-molybdate, calculated from the regression, is 1.0037 . From this value, we calculate that the ratio of efflux to influx is about $0 \cdot 19$. That is, in the absence of tungstate, more than $80 \%$ of $\mathrm{NO}_{3}^{-}$entering the cell undergoes reduction with less than $20 \%$ re-entering the medium from the cell, even when the molybdate concentration was decreased by a factor of 10 below the normal concentration. With the normal molybdate concentration $(1.8 \mu \mathrm{M})$, the value of $\beta_{\mathrm{obs}}$ was 1.0003 , implying that more than $99 \%$ of the $\mathrm{NO}_{3}^{-}$ entering the cell undergoes reduction.

The small difference in $\beta_{\mathrm{obs}}$ for the two molybdate concentrations ( 1.8 and $0.18 \mu \mathrm{M})$ (Fig. $3 b)$ is consistent with the NR concentration being limiting at the lower molybdate concentration. In this case, one might have expected a lower value of $k$ for the lower molybdate concentration. This was not observed (Fig. 3a). However, there was no correlation between $k$ and $\beta_{\mathrm{obs}}$ in replicate experiments (Table 1), suggesting an unknown variable causing differences in $k$ but which does not affect the ratio of efflux to influx. It seems possible that a difference in $k$ caused by NR concentration might be offset by such an unknown variable.

We thank Professor Jane Gibson (Cornell University, Ithaca, NY, USA) who graciously shared her extensive experience and knowledge of $\mathrm{N}$ metabolism of cyanobacteria, and encouraged us to believe that it would be useful to be able to tease apart the influx and efflux components of net $\mathrm{NO}_{3}^{-}$uptake in a steady-state system. We also thank her for extending the hospitality of her laboratory to one of us (G.S.) in order to learn a few of the tricks of working with cyanobacteria. The comments of two reviewers were appreciated, and resulted in a much improved manuscript. The work reported here was funded by the United States Department of Energy, grant no. DE-FG0287ER13765. A000.

\section{References}

Bergersen, F. (1980). Measurement of nitrogen fixation by direct means. In Methods for Evaluating Biological Nitrogen Fixation, pp. 235-252. Edited by F. Bergersen. New York: John Wiley.

BREMNER, J. M. \& KeENEY, D. R. (1965). Steam distillation methods for determination of ammonium, nitrate and nitrite. Analytica Chimica Acta 32, 485-495.

Candau, P. (1979). Purificacion y propiedades de la ferredoxina-nitrato reductasa de la cianobacteria Anacystis nidulans. PhD dissertation. Universidad de Sevilla, Savilla, Spain.

Candau, P., Manzano, C. \& Losada, M. (1976). Bioconversion of light energy. Nature, London 262, 715-717.

Flores, E., Guerrero, M. G. \& Losada, M. (1980). Short term ammonium inhibition of nitrate utilization by Anacystis nidulans and other cyanobacteria Archives of Microbiology 128, 137-144.

Herrero, A., Flores, E. \& Guerrero, M. G. (1981). Regulation of nitrate reductase in the cyanobacterium Anacystis nidulans, Anabaena sp. strain 7119, and Nostoc sp. strain 6719. Journal of Bacteriology 145, 175-180.

KRÄMER, E. \& SCHMIDT, A. (1989). Nitrite accumulation by Synechococcus 6301 as a consequence of carbon- or sulfur-deficiency. Federation of European Microbiology Society Letters 59, 191-196.

Lara, C. \& Romero, J. (1986). Distinctive light and $\mathrm{CO}_{2}$-fixation requirements of nitrate and ammonium utilization by the cyanobacterium Anacystis nidulans. Plant Physiology 81, 686-688.

Lara, C., Romero, J. \& Guerrero, M. G. (1987). Regulated nitrate transport in the cyanobacterium Anacystis nidulans. Journal of Bacteriology 169, 4376-4378.

Madueño, F., Borrias, W. E., Van Arkel, G. A. \& Guerrero, M. G. (1988). Isolation and characterization of Anacystis nidulans R2 mutants affected in nitrate assimilation: establishment of two new mutant types. Molecular and General Genetics 213, 223-228.

Mariotti, A., Germon, J. C., Hubert, P., Kaiser, P., Letolle, R., TARDIEUX, A. \& TARDieuX, P. (1981). Experimental determination of nitrogen kinetic isotope fractionation: some principles; illustrations for the denitrification and nitrification processes. Plant and Soil 62, 413-430.

Melander, L. \& SaUnders, W. H. J. (1980). Reaction Rates of Isotopic Molecules. New York:, John Wiley.

Rippka, R., Derruelles, J., Waterbury, B., Herdman, M. \& StaniER, R. Y. (1979). Generic assignments, strain histories and properties of pure cultures of cyanobacteria. Journal of General Microbiology 111, 1-61

Romero, J. M., Karam, C. \& Guerrero, M. G. (1985). Dependence of nitrate utilization upon active $\mathrm{CO}_{2}$ fixation in Anacystis nidulans: a regulatory aspect of the interaction between photosynthetic carbon and nitrogen metabolism. Archives of Microbiology 142, 394-401.

SHEARER, G. \& KOHL, D. H. (1986). $\mathrm{N}_{2}$-fixation in field settings: estimations based on natural ${ }^{15} \mathrm{~N}$ abundance. Australian Journal of Plant Physiology 13, 699-756.

Shearer, G. \& KoHL, D. H. (1988). Nitrogen isotopic fractionation and ${ }^{18} \mathrm{O}$ exchange in relation to the mechanism of denitrification by Pseudomonas stutzeri. Journal of Biological Chemistry 263, 1323113245. 